\title{
Caracterización de la secuencia del gen de la proteín transporta- dora de péptidos (PepT1) en alevines de paiche Arapaima gigas
}

\section{Characterization of the gene sequence of the peptide transport protein (PepT1) in paiche Arapaima gigas fingerlings}

\author{
Odalis E. Toledo ${ }^{1,2,4}$, Arnaldo E. Castañeda ${ }^{1,2}$, Manuel A. Feriaa, \\ Pedro Masías ${ }^{2}$, Mario Cueva ${ }^{3}$, Emmerik Motte $^{2}$
}

\section{Resumen}

\begin{abstract}
El objetivo del estudio fue caracterizar el PepT1 del paiche Arapaima gigas mediante herramientas genómicas y proteómicas. Se realizó un análisis transcriptómico y proteómico a partir de secciones del intestino, bazo, hígado y riñón de alevines. El análisis transcriptómico permitió obtener dos secuencias consenso de 357 y 459 pb. Ambos fragmentos presentaron un alto grado de homología (78 y 80\%), principalmente con secuencias de nucleótidos codificantes del PepT1 de Scleropages formosus del mismo Orden que el paiche. El análisis proteómico por espectrometría de doble masa MALDI TOF/TOF permitió identificar quince secuencias peptídicas del PepT1 en paiche. Como conclusión se logró caracterizar parcialmente el PepT1 en el intestino del paiche, las cuales podrán ser usadas para posteriores estudios sobre la evaluación de su expresión.
\end{abstract}

Palabras clave: Arapaima gigas; proteína transportadora de péptido; PepT1; proteómica; espectrometría de masas; MALDI TOF/TOF

\section{Abstract}

The aim of this study was to characterize the PepT1 of pirarucu Arapaima gigas through genomic and proteomic tools. A transcriptomic and proteomic analysis was made from sections of the intestine, spleen, liver and kidney of fingerlings. The transcriptomic analysis allowed obtaining two consensus sequences of 357 and $459 \mathrm{bp}$. Both fragments showed a high degree of homology (78 and 80\%), mainly with nucleotide

\footnotetext{
${ }^{1}$ Facultad de Pesquería, Universidad Nacional de Tumbes, Tumbes, Perú

${ }^{2}$ Empresa de Formación e Investigación en Biotecnología Molecular, INCABIOTEC SAC, Tumbes, Perú

${ }^{3}$ Cooperativa de Trabajadores Biotecoop, Tumbes, Perú

${ }^{4}$ E-mail:Odat68@gmail.com
}

Recibido: 2 de noviembre de 2018

Aceptado para publicación: 28 de mayo de 2019 
sequences coding for the PepT1 of Scleropages formosus of the same Order as A. gigas. The proteomic analysis by double mass spectrometry MALDI TOF/TOF allowed to identify 15 peptide sequences of PepT1 in pirarucu. In conclusion, PepT1 was partially characterized in the intestine of pirarucu, which could be used for further studies on the evaluation of its expression.

Key words: Arapaima gigas; peptide transporter; PepT1; proteomic; mass spectrometry; MALDI TOF/TOF

\section{INTRODUCCIÓN}

El paiche Arapaima gigas (Cuvier, 1829), es una especie importante de la ictiofauna amazónica que pertenece a la familia Osteoglossidae. Se caracteriza por su elevada tasa de crecimiento (10-15 kg por año) y excelente sabor de su carne (Rebaza et al., 2010), convirtiéndose en una especie con gran potencial en la acuicultura peruana. No obstante, una de las principales limitantes para su producción sostenible e intensiva es la nutrición (Oeda et al., 2015), de allí que se realizan estudios sobre la tasa de ingestión y digestión para el desarrollo y formulación de dietas, utilizando fuentes alternas de nutrientes (DIDIT, 2017).

Los peces, en especial los alevinos, requieren de altas cantidades de proteínas que, asimiladas de manera eficiente una vez reducidas a dipéptidos y tripéptidos, son empleados para su crecimiento y fuente de energía (Gatlin et al., 2007; Verri et al., 2011; Liu et al., 2013). Las proteínas son sometidas a un proceso de digestión en el estómago, cuyos productos ingresan al intestino delgado para ser degradados por las enzimas pancreáticas generando productos como aminoácidos, oligopéptidos absorbibles y ditri péptidos (Kiela y Grishan, 2016), que son llevados por proteínas transportadores de péptidos (Verri et al., 2011) a través de la membrana celular de los enterocitos (Gilbert et al., 2008).
El péptido transportador 1 (PepT1), categorizado como miembro de la familia 15 , portadoras de soluto A1 (SLC15A1), es el principal conductor de nutrientes debido a su transporte no selectivo y alta capacidad de función para tri y di péptidos a partir del lumen intestinal hacia el interior de los enterocitos (Segura et al., 2010). El PepT1 se localiza principalmente en el borde de cepillo de las células epiteliales del intestino (Wang et al., 2017), posee un tamaño de aproximadamente 700 aminoácidos ( $\approx 2100$ nucleótidos) en vertebrados superiores, mientras que en la mayoría de los vertebrados inferiores es usualmente más grande (Ostaszewska et al., 2010). Sin embargo, la secuencia de aminoácidos como de nucleótidos son altamente conservadas en comparación con los vertebrados superiores (Ronnestad et al., 2014). Además, posee 12 dominios transmembrana, con los terminales carboxilo y amino en el sitio citoplasmático (Daniel, 2004), y dos isoformas: PepT1a y PepT1b (Bucking y Schulte, 2012).

E1 PepT1 se expresa principalmente en el intestino anterior (Ronnestad et al., 2007; Bakke et al., 2010), pero también ha sido detectado en otros tejidos como branquias, cerebro, corazón, hígado, bazo y riñón en niveles muy bajos (Wang, 2017). Además, es regulada por una variedad de hormonas como factores de crecimiento epidermal, ritmo diurno y principalmente por el contenido de proteína en la dieta (Liu et al., 2013). Estudios sobre el Pept1 se han dirigido principalmente 
a su secuencia, estructura, función, expresión, lugar de distribución (tejido/órgano), y factores ambientales en diferentes variedades de especies de peces (Bakke et al., 2010), tales como Anguilla anguilla (Storelli et al., 1989; Thamotharan et al., 1996), pez zebra Danio rerio (Verri et al., 2003), carpa común Cyprinus carpio (Ostaszewska et al., 2009), salmón del atlántico Salmo salar (Ronnestad et al., 2010) y trucha Oncorhynchus mykiss (Ostaszewska et al., 2010). Sin embargo, hasta la fecha, no existe investigaciones sobre la caracterización del PepT 1 en el tracto gastrointestinal del paiche A. gigas.

Por lo tanto, el objetivo de este estudio fue caracterizar molecularmente la proteína transportadora de péptidos (PepT1) del intestino de paiche $A$. gigas mediante técnicas genómicas y proteómicas, para generar una base de datos para posteriores estudios sobre la regulación de su expresión, y el potencial en la formulación de alimentos para el paiche.

\section{MATERIALeS y Métodos}

\section{Alineamiento de Secuencias y Diseño de Cebadores}

Se recopilaron 16 secuencias de ADNc del PepT1 de teleósteos evolutivamente cercanos con el paiche (Cuadro 1), a partir de la base de datos del Centro Nacional de Información Biotecnológica NCBI (www.ncbi.nlm.nih.gov/). La homología entre secuencias se analizó mediante Basic Local Aligment Search Tool (BLAST) y el alineamiento múltiple mediante el programa Clustal W (http://www.ebi.ac.uk/Tolls/msa/ clustalw2/). A partir de la secuencia conservada se realizó el diseño de los iniciadores degenerados (Cuadro 2), los cuales fueron sintetizados por la empresa Integrated DNA Technologies (IDT, USA).

\section{Muestras y Preparación de Tejidos}

Se utilizaron 15 alevines de paiche de aproximadamente $15 \mathrm{~cm}$ de longitud, obtenidos de una empresa local, ubicada en el departamento de Tumbes, Perú. Los peces fueron eutanasiados, previa sedación con eugenol (Acuestrol ${ }^{\circledR}$ ) en solución al 1\% del stock. Se realizó una apertura en la cavidad abdominal y se hizo un raspado del epitelio intestinal de las regiones anterior, medio y posterior. Además, se colectaron muestras de riñón, hígado y bazo $(<0.5 \mathrm{~cm})$. Los tejidos fueron preservados en RNAlater (Ambion, Austin, TX, USA) y se almacenaron a $-20{ }^{\circ} \mathrm{C}$ hasta su análisis.

\section{Extracción de ARN Total y Síntesis de ADNc}

La extracción de ARN total de los tejidos se realizó con el kit GeneJET RNA Purification (Thermo Fisher Scientific, USA) de acuerdo a las instrucciones del fabricante. La cadena de ADNc fue sintetizada utilizando el kit ReverdtAid ${ }^{\mathrm{TM}}$ Reverse Transcriptase (Fermentas ${ }^{\circledR}$ ) en presencia del iniciador Oligo dT1 18 en una reacción de 20 $\mu 1$, siguiendo el protocolo recomendado por el fabricante y sujeto a tratamiento con DNase (DNase I, Thermo Fisher Scientific).

\section{Optimización de PCR para Iniciadores Degenerados}

El ADNc fue amplificado en volúmenes de $25 \mu$, conteniendo 0.04 Unidades de Hot Start Taq DNA polimerasa (Thermo Fischer Scientific), solución de tampón de la polimerasa a $1 \mathrm{X}, 2 \mathrm{mM}$ de $\mathrm{MgCl}, 0.2 \mathrm{mM}$ de cada dNTPs, 0.6 y $1.2 \mu \mathrm{M}$ para los iniciadores degenerados y el control interno $18 \mathrm{~S}$ rRNA, respectivamente, ADNc y agua libre de nucleasas (AUP). Se determinó la temperatura de hibridación óptima para cada juego de iniciadores de acuerdo con las temperaturas de fusión (melting) (Cuadro 3) y los productos amplificados fueron separados por 
Cuadro 1. Número de acceso de las secuencias del ADNc del PepT1 de peces

\begin{tabular}{llcc}
\hline $\mathrm{N}^{\circ}$ de accesión & Especie & ARNm $(\mathrm{pb})$ & Aminoácidos (aa) \\
\hline EU160494.1 & Sebastes nebulosus & 2640 & 742 \\
NM_198064.1 & Danio rerio & 2637 & 718 \\
JN896885.1 & Cyprinus carpio & 3118 & 723 \\
JN088166.1 & Ctenopharyngodon idella & 2761 & 713 \\
AB762417.1 & Anguilla japónica & 2952 & 723 \\
AB455540.1 & Salmo salar & 2629 & 734 \\
JN615007.1 & Fundulus heteroclitus & 2807 & 722 \\
KC476396.1 & Cynoglossus semilaevis & 2684 & 729 \\
LC167343.1 & Oreochromis mossambicus & 2787 & 727 \\
AY170828.2 & Chionodraco hamatus & 2845 & 757 \\
KC485004.1 & Larimichthys crocea & 2923 & 726 \\
GQ906471.2 & Perca flavescens & 2956 & 729 \\
XM_018729534.1 & Scleropages formosus & 2046 & 676 \\
FJ237043.2 & Dientrarchus labrax & 2184 & 728 \\
AY921634.1 & Gadus morhua & 3014 & 727 \\
JX177494.1 & Tetraodon nigroviridis & 2328 & 725 \\
\hline
\end{tabular}

Cuadro 2. Iniciadores usados para la transcriptasa inversa-PCR del ADNc del PepT1 en paiche (Arapaima gigas)

\begin{tabular}{|c|c|c|c|}
\hline Iniciador & Secuencia 5'-3' & $\operatorname{Tm}\left({ }^{\circ} \mathrm{C}\right)$ & $\begin{array}{c}\text { Tamaño } \\
(\mathrm{pb})\end{array}$ \\
\hline $18 S r R N A F^{1}$ & TACCACATCCAAAGAAGGCAG & 54.9 & \multirow{2}{*}{200} \\
\hline $18 S r R N A R^{1}$ & TCGATCCCGAGATCCAACTAC & 57.5 & \\
\hline PepT1 F2² & TCCCHCTHCCIATGTTCTGG & 58.6 & \multirow{2}{*}{914} \\
\hline PepT1 R3 $3^{2}$ & GCCTGTGARTAIGMGAACTCCA & 62 & \\
\hline Pept $1 F 3^{2}$ & TTCTCCTACTATGGCATGMRAGC & 61 & \multirow{2}{*}{697} \\
\hline PepT1 R1 $1^{2}$ & IGCCCAGTCCATCCAGT & 59 & \\
\hline Pept1 F4 $4^{2}$ & TGGCTSGGIAAGTTCAAGAC & 59 & \multirow{2}{*}{550} \\
\hline $\operatorname{PepTIR} 1^{2}$ & IGCCCAGTCCATCCAGT & 59 & \\
\hline
\end{tabular}

${ }^{1}$ Referencia: Regina et al. (2014)

${ }^{2}$ Referencia: este estudio 
Cuadro 3. Perfil térmico para la amplificación de regiones del PepT 1

\begin{tabular}{|c|c|c|c|c|c|c|}
\hline & $\begin{array}{l}0 \\
0 \\
0 \\
0 \\
0 \\
0 \\
0\end{array}$ & 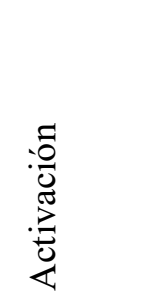 & 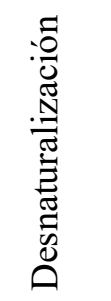 & 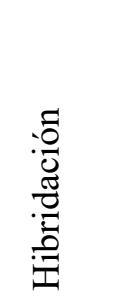 & 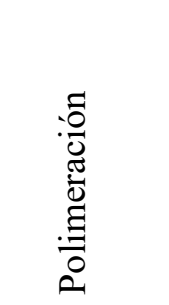 & 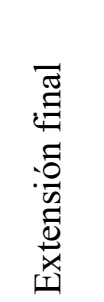 \\
\hline & Ciclos & & & 35 & & \\
\hline \multirow[t]{5}{*}{ A } & Temp. & $95^{\circ} \mathrm{C}$ & $95^{\circ} \mathrm{C}$ & $54{ }^{\circ} \mathrm{C}$ & $72^{\circ} \mathrm{C}$ & $72^{\circ} \mathrm{C}$ \\
\hline & & & & $55^{\circ} \mathrm{C}$ & & \\
\hline & & & & $56^{\circ} \mathrm{C}$ & & \\
\hline & & & & $57^{\circ} \mathrm{C}$ & & \\
\hline & Tiempo & $5 \mathrm{~min}$ & $45 \mathrm{~s}$ & $30 \mathrm{~s}$ & $45 \mathrm{~s}$ & $5 \mathrm{~min}$ \\
\hline \multirow[t]{6}{*}{ B } & Ciclos & & & 35 & & \\
\hline & Temp. & $95^{\circ} \mathrm{C}$ & $95^{\circ} \mathrm{C}$ & $54^{\circ} \mathrm{C}$ & $72^{\circ} \mathrm{C}$ & $72^{\circ} \mathrm{C}$ \\
\hline & & & & $55^{\circ} \mathrm{C}$ & & \\
\hline & & & & $56^{\circ} \mathrm{C}$ & & \\
\hline & & & & $57^{\circ} \mathrm{C}$ & & \\
\hline & Tiempo & $5 \mathrm{~min}$ & $45 \mathrm{~s}$ & $45 \mathrm{~s}$ & $1 \min 30 s$ & $5 \mathrm{~min}$ \\
\hline \multirow[t]{6}{*}{$\mathrm{C}$} & Ciclos & & & 35 & & \\
\hline & Temp. & $95^{\circ} \mathrm{C}$ & $95^{\circ} \mathrm{C}$ & $55^{\circ} \mathrm{C}$ & $72^{\circ} \mathrm{C}$ & $72{ }^{\circ} \mathrm{C}$ \\
\hline & & & & $56^{\circ} \mathrm{C}$ & & \\
\hline & & & & $57^{\circ} \mathrm{C}$ & & \\
\hline & & & & $58^{\circ} \mathrm{C}$ & & \\
\hline & Tiempo & $5 \mathrm{~min}$ & $30 \mathrm{~s}$ & $45 \mathrm{~s}$ & $1 \mathrm{~min}$ & $5 \mathrm{~min}$ \\
\hline
\end{tabular}

A: Ciclos térmicos para amplificar de 18 sRNA F, R (200 pb); B: Ciclos térmicos para amplificar Pept1 F2, R3 (914 pb); C: Ciclos térmicos para amplificar Pept1 F3, R1 (697)

migración electroforética en gel de agarosa al $2 \%$, teñido con bromuro de etidio y sumergido en una solución TAE $1 \mathrm{X}$, para ser revelado en un transluminador. Los productos amplificados fueron secuenciados por la empresa Macrogen (USA).

\section{Análisis in silico}

Las secuencias de nucleótidos se analizaron con el programa CodonCode Aligner. Las secuencias de aminoácidos fueron predichas utilizando lo programas ExPASy
Translate Tool (http://web.expasy.org/ translate/).

\section{Extracción de Proteínas y Migración en Gel de Poliacrilamida}

En la extracción de proteínas intestinales se utilizó el reactivo Trizol ${ }^{\circledR}$ (Invitrogen, Carlsbad, USA), siguiendo el protocolo del fabricante. Las proteínas extraídas fueron separadas por electroforesis en gel de dodecil sulfato de sodio y policrilamida (SDS-PAGE) mediante un sistema vertical mini OmniPAGE 
(Cleaver Scientific ${ }^{\circledR}$, Wrwickshire, UK) durante $3 \mathrm{~h}$ a $90 \mathrm{v}$ al $12 \%$ de gel.

\section{Purificación y Digestión de Péptidos}

Las bandas fueron extraídas y digeridas siguiendo el protocolo descrito por Shevchenko et. al. (2007) modificado. Las muestras fueron hidratadas con agua grado HPLC con TFA y se les adicionó el mismo volumen de matriz R-cyano-4 hydroxycynnamic (á-CHCA, Sigma Aldrich) $(10 \mathrm{mg} / \mathrm{ml})$ $(\mathrm{V} / \mathrm{V})$, se homogenizaron cuidadosamente $\mathrm{y}$ fueron colocadas en el spot de la Opti TOF® placa MALDI.

\section{Análisis por MALDI TOF/TOF}

La identificación de los péptidos se realizó con un espectrómetro de masas MALDI TOF/TOF 5800 (Applied Biosystems, SCIEX) en modo ion reflector positivo, con una intensidad de láser Nd:YAG de $\lambda$ a 335 $\mathrm{nm}$, frecuencia $200 \mathrm{~Hz}, 750$ disparos por espectro y analizados dentro de un rango de masas entre 800 a $4000 \mathrm{~m} / \mathrm{z}$. Los péptidos obtenidos del procesamiento por doble fragmentación (MS/MS) fueron analizados mediante el software ProteinPilot 4.0. Las secuencias obtenidas fueron alineadas utilizando la base de dato libre NCBI Blast a través de la plataforma protien blast.

\section{Resultados}

\section{PCR del PepT1 de Paiche}

Todas las temperaturas de hibridación y los ciclos térmicos utilizados resultaron en productos del tamaño esperado para cada juego de iniciadores, excepto el último iniciador (Cuadro 2). Se observaron bandas definidas de $200 \mathrm{pb}$ para los iniciadores controles 18S ARNr F y 18S ARNr R (Figura 1a). Se observaron bandas definidas de $697 \mathrm{pb}$ para los iniciadores degenerados PepT1 F3 y PepT1 R1 en todas las temperaturas hibridación probadas (Figura 1b). También se ob- servó hibridación para los iniciadores degenerados PepT1 F2 y PepT1 R3 mostrando un producto de $914 \mathrm{pb}$ (Figura 1c).

\section{Identificación del PepT1 en Tejidos}

Se observaron bandas definidas correspondientes a productos de $914 \mathrm{pb}$, que fueron amplificados a partir del aislamiento de ARNm del intestino anterior, medio y posterior. No hubo presencia del producto en bazo, hígado y riñón (Figura 2).

\section{Análisis Bioinformático de las Secuen- cias}

Los fragmentos de ADNc del PepT1 del paiche fueron secuenciados, analizados y traducidos en sus secuencias de aminoácidos potenciales. Se determinaron dos secuencias consenso de tamaño de $357 \mathrm{pb}$ (118 aa) y 459 $\mathrm{pb}$ (152 aa), como se muestra en la Figura 3.

Las secuencias parciales de nucleótidos exhibieron una alta similitud de secuencia con el PepT1 del Scleropages formosus (GenBank XM_018729534.1). En particular, el primer fragmento del gen de ADNc del PepT1 que exhibió un $84 \%$ de similitud con el sfPepT1, y de aproximadamente $78 \%$ de similitud con los demás teleósteos. Similarmente, el segundo fragmento exhibió un $73 \%$ de similitud con el sfPepT1. Además, las secuencias de aminoácidos predichas a partir de los fragmentos exhibieron una similitud de $81 \%$ con la secuencia de aminoácido del sfPepT1 (Cuadro 4).

\section{Identificación por MALDI TOF/TOF PepT1}

El análisis por MALDI TOF/TOF mostró un total de 15 secuencias correspondientes al Pept1, en particular las secuencias IR N I K F G D V VA K P EE GA N A IR, NVIISYGNETTNELLNVMGK y TYEIFKDIKTKPEEGANAIR por el nivel de identidad $100 \%$ y un valor de E. de $3 \mathrm{e}^{-12}$, al ser comparada con la secuencia de Poecilia 
Cuadro 4. Similitud de las secuencias del gen del PepT1 del paiche frente a otras secuencias del gen de PepT1 de peces con respecto a los nucleótidos y aminoácidos que codifican la proteína del PepT1 (Blast)

\begin{tabular}{lcccc}
\hline & \multicolumn{2}{c}{$\begin{array}{c}\text { Fragmento 1 del PepT1 de } \\
\text { paiche }\end{array}$} & \multicolumn{2}{c}{$\begin{array}{c}\text { Fragmento 2 del PepT1 de } \\
\text { paiche }\end{array}$} \\
\cline { 2 - 6 } Especies & $\begin{array}{c}\text { Similitud de } \\
\text { nucleótidos } \\
(\%)\end{array}$ & $\begin{array}{c}\text { Similitud de } \\
\text { aminoácidos } \\
(\%)\end{array}$ & $\begin{array}{c}\text { Similitud de } \\
\text { nucleótidos } \\
(\%)\end{array}$ & $\begin{array}{c}\text { Similitud de } \\
\text { aminoácidos } \\
(\%)\end{array}$ \\
\hline Scleropages formosus & 84 & 81 & 73 & 55 \\
Anguilla japonica & 77 & 78 & ---- & 42 \\
Oncorhynchus tshawytscha & 79 & 83 & ---- & 44 \\
Lates calcarifer & 80 & 83 & ---- & 39 \\
Ictalurus punctatus & 75 & 82 & ---- & 42 \\
Larimichthys crocea & 76 & 80 & --- & 44 \\
Esox lucius & 77 & 82 & ---- & 43 \\
Oreochromis mossambicus & 77 & 80 & ---- & 42 \\
Oreochromis niloticus & 76 & 80 & ---- & 42 \\
Danio rerio & ---- & & ---- & 42 \\
Cyprinus carpio & ---- & 74 & ---- & 41 \\
Salmo salar & 79 & 83 & ---- & 44 \\
Tetraodon nigroviridis & 75 & ------ & --- & ---- \\
\hline
\end{tabular}

reticulata, Pelodiscus sinensis y Paralichthys olivaceus, respectivamente, de la base de datos de Protein BLAST. Los resultados resumidos se presentan en el Cuadro 5.

\section{Discusión}

La proteína transportadora de péptidos (PepT1) es responsable de la absorción de aminoácidos y oligopéptidos en el intestino, siendo importante en el crecimiento de peces (Verri et al., 2011; Romano et al., 2014); además de ser confirmada como marcador del metabolismo, especialmente de las proteínas (Liu, 2014), habiendo sido identificada en varias especies; sin embargo, su presencia en el paiche $A$. gigas era desconocida. En el presente estudio se logró identificar por primera vez las secuencias nucleotídicas parciales de la proteína transportadora PepT1 del intestino de esta especie.
En el presente estudio, se optimizaron los iniciadores degenerados PepT1 F3, PepT1 R1 y PepT1 F2, PepT1 R3, apreciándose bandas definidas a todas las temperaturas probadas. Esto se explica porque las temperaturas escogidas se encontraban $5{ }^{\circ} \mathrm{C}$ por encima o debajo de la temperatura de fusión (Tm). Así mismo, se sabe que temperaturas muy altas causan un mal alineamiento entre iniciadores provocando poco ADN amplificado y temperaturas muy bajas causan la amplificación de ADN inespecífico (Sambrook y Russell, 2001). Por otro lado, se consideró el uso de 18S ARNr como control interno para asegurar la correcta extracción de ARN y PCR de la muestra y así evitar los falsos negativos. Estudios indican que entre los controles internos más utilizados se encuentran el EFA, B-actina, GAPDH y $18 \mathrm{~S}$ ARNr siendo este último el menos variable (Fernandes et al., 2008; Su et al., 2011). 
Cuadro 5. Secuencias de aminoácidos identificadas por MALDI TOF/TOF (Protein BLAST)

\begin{tabular}{|c|c|c|c|c|c|}
\hline Prec MW & Prec M Z & Secuencia de aminoácido & Especie & Ident & $\mathrm{N}^{\circ}$ Accesión \\
\hline $\begin{array}{c}1197.712 \\
6\end{array}$ & $\begin{array}{c}1198.712 \\
6\end{array}$ & AIKNRFRHR & $\begin{array}{l}\text { Poecilia } \\
\text { reticulata }\end{array}$ & 100 & $\begin{array}{l}\text { XP_008396824. } \\
1\end{array}$ \\
\hline $\begin{array}{c}1331.664 \\
7\end{array}$ & $\begin{array}{c}1332.664 \\
7\end{array}$ & LCRINFTPLR & Danio rerio & 100 & NP_932330.1 \\
\hline 999.4227 & $\begin{array}{c}1000.422 \\
7\end{array}$ & TLAALGQKR & $\begin{array}{l}\text { Alligator } \\
\text { mississippiensis }\end{array}$ & 100 & $\begin{array}{l}\text { XP_014455978. } \\
1\end{array}$ \\
\hline $\begin{array}{c}1318.675 \\
7\end{array}$ & $\begin{array}{c}1319.675 \\
7\end{array}$ & CIGFAIKNRIR & $\begin{array}{l}\text { Poecilia } \\
\text { reticulata }\end{array}$ & 91 & $\begin{array}{l}\text { XP_008396824. } \\
1\end{array}$ \\
\hline $\begin{array}{c}1353.631 \\
7\end{array}$ & $\begin{array}{c}1354.631 \\
7\end{array}$ & DWAKEKYDKR & $\begin{array}{l}\text { Rhincodon } \\
\text { typus }\end{array}$ & 88 & $\begin{array}{l}\text { XP_020367844. } \\
1\end{array}$ \\
\hline $\begin{array}{c}1514.763 \\
7\end{array}$ & $\begin{array}{c}1515.763 \\
7\end{array}$ & FCERFSYYGMR & Esox lucius & 100 & $\begin{array}{l}\text { XP_012993296. } \\
1\end{array}$ \\
\hline $\begin{array}{c}1217.525 \\
6\end{array}$ & $\begin{array}{c}1218.525 \\
6\end{array}$ & AVALVVFISGSR & $\begin{array}{l}\text { Ictalurus } \\
\text { punctatus }\end{array}$ & 100 & $\begin{array}{l}\text { XP_017337526. } \\
1\end{array}$ \\
\hline $\begin{array}{c}1197.712 \\
6\end{array}$ & $\begin{array}{c}1198.712 \\
6\end{array}$ & AENGGTQTRPR & $\begin{array}{l}\text { Paralichthys } \\
\text { olivaceus }\end{array}$ & 100 & $\begin{array}{l}\text { XP_019960053. } \\
1\end{array}$ \\
\hline $\begin{array}{c}1633.763 \\
7\end{array}$ & $\begin{array}{c}1634.763 \\
7\end{array}$ & TQNQEFNITPVDVR & $\begin{array}{l}\text { Rhincodon } \\
\text { typus }\end{array}$ & 100 & $\begin{array}{l}\text { XP_020367844. } \\
1\end{array}$ \\
\hline $\begin{array}{c}1814.958 \\
6\end{array}$ & $\begin{array}{c}1815.958 \\
6\end{array}$ & KNMEKDKMGMAIESR & $\begin{array}{l}\text { Nothobranchiu } \\
\text { s furzeri }\end{array}$ & 100 & $\underset{1}{\text { XP_015807884. }}$ \\
\hline $\begin{array}{c}1789.936 \\
6\end{array}$ & $\begin{array}{c}1790.936 \\
6\end{array}$ & WTLQATTMNGQIGLLK & Labrus bergylta & 100 & $\begin{array}{l}\text { XP_020500572. } \\
1\end{array}$ \\
\hline $\begin{array}{c}2179.145 \\
8\end{array}$ & $\begin{array}{c}2180.145 \\
8\end{array}$ & QPEHGPERKRDQVEMER & Salmo salar & 100 & $\begin{array}{l}\text { NP_001140154. } \\
1\end{array}$ \\
\hline $\begin{array}{c}2322.107 \\
7\end{array}$ & $\begin{array}{c}2323.107 \\
7\end{array}$ & TYEIFKDIKTKPEEGANAIR & $\begin{array}{l}\text { Paralichthys } \\
\text { olivaceus }\end{array}$ & 100 & $\begin{array}{l}\text { XP_019960053. } \\
1\end{array}$ \\
\hline $\begin{array}{c}2227.095 \\
7\end{array}$ & $\begin{array}{c}2228.095 \\
7\end{array}$ & NVIISYGNETTNELLNVMGK & $\begin{array}{l}\text { Pelodiscus } \\
\text { sinensis }\end{array}$ & 100 & $\begin{array}{l}\text { XP_006128192. } \\
1\end{array}$ \\
\hline $\begin{array}{c}2322.107 \\
7\end{array}$ & $\begin{array}{c}2323.107 \\
7\end{array}$ & $\begin{array}{l}\text { IRNIKFGDVVAKPEEGANAI } \\
\mathrm{R}\end{array}$ & $\begin{array}{l}\text { Poecilia } \\
\text { formosa }\end{array}$ & 100 & $\begin{array}{l}\text { XP_007565446. } \\
1\end{array}$ \\
\hline
\end{tabular}

Se determinó la presencia del ARN mensajero del PepT1 en intestino (anterior, medio y posterior) del paiche evidenciada en bandas del tamaño de $914 \mathrm{pb}$; sin embargo, no se identificó en riñón, hígado y bazo. Resultados similares fueron presentados por Verri et al. (2011), quienes indican que la distribución del PepT1 en peces teleósteos se da principalmente en el intestino y en menor grado en riñón, hígado, bazo y otros órganos o tejidos, pudiendo diferir significativamente de un grupo de peces a otro. Además, se pue- de observar una disminución de intensidad de bandas a lo largo del segmento del intestino (del anterior al posterior), pudiendo diferir con otros estudios que indican que la expresión del PepT1 disminuye a lo largo del segmento proximal al distal del lumen intestinal, representando la diferencia entre los segmentos en la absorción de proteínas (Shen et al., 2001; Terova et al., 2009; Ostaszewska et al., 2010). Sin embargo, estudios cuantitativos basados en qPCR en tiempo real son necesarios para completar el estudio. 
A.

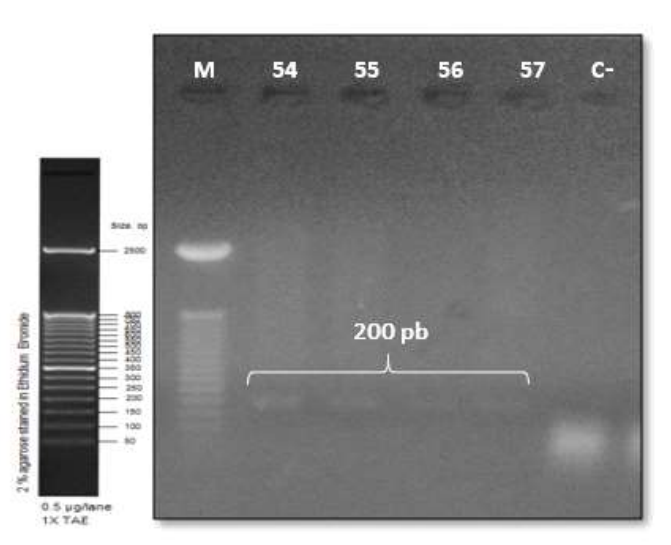

B.

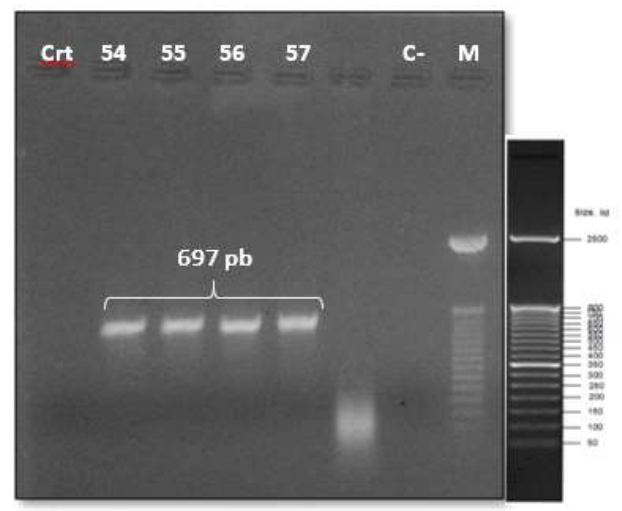

C.

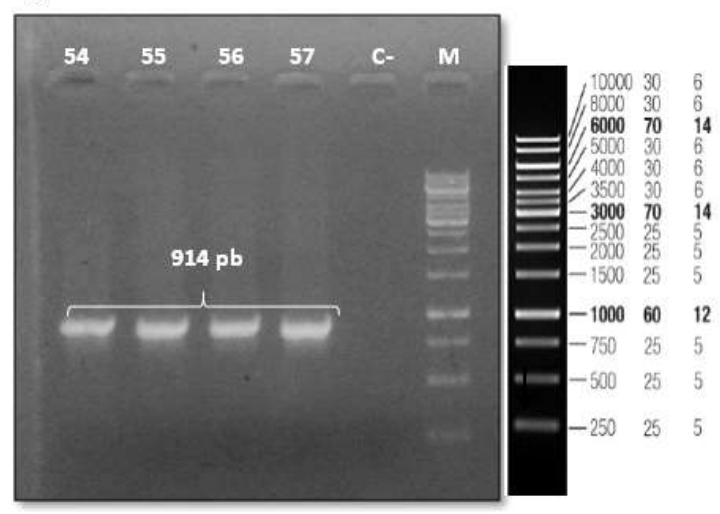

Figura 1. Migración en gel de agarosa de los productos obtenidos por PCR. A. Productos de la amplificación con iniciadores $18 \mathrm{~s} \mathrm{ARNr}(\mathrm{F}, \mathrm{R})$ para la determinación de la temperatura de hibridación. M: marcador de peso molecular de $50 \mathrm{pb}$ (Invitrogen), 54, 55, 56 y 57: Muestras amplificadas a diferentes temperaturas de hibridación, C-: control negativo. B. Productos de la amplificación con iniciadores degenerados PepT1 F3 y PepT1 R1. Crt: Control de la transcriptasa reversa; 54, 55, 56, 57: Muestras amplificadas a diferentes temperaturas de hibridación; M: Marcador de peso molecular de $50 \mathrm{pb}$ (Invitrogen), C: control negativo. C. Productos de amplificación con los iniciadores degenerados PepT1 F2 y PepT1 R3. 54, 55, 56, 57: Muestras amplificadas a diferentes temperaturas de hibridación; M: marcador de peso molecular de $1 \mathrm{~Kb}$ (Thermo Fisher); C-: control negativo

Las secuencias consenso del fragmento 1 y 2 mostraron una identidad con la secuencia del ARNm del PepT1 del Scleropages formosus de 84 y $73 \%$, respectivamente. Esto demuestra una alta similitud de nucleótidos con el paiche, confirmando de esta manera su parentesco familiar Osteoglossidae. Además, estos datos consti- tuyen una primera evidencia de la presencia del producto de ARNm relacionado con el PepT1 en la mucosa intestinal en el paiche.

La proteómica busca estudiar las proteínas totales presentes en una célula, tejido o fluido corporal en un momento dado, ya sea por abundancia, actividad, estructura, estado 


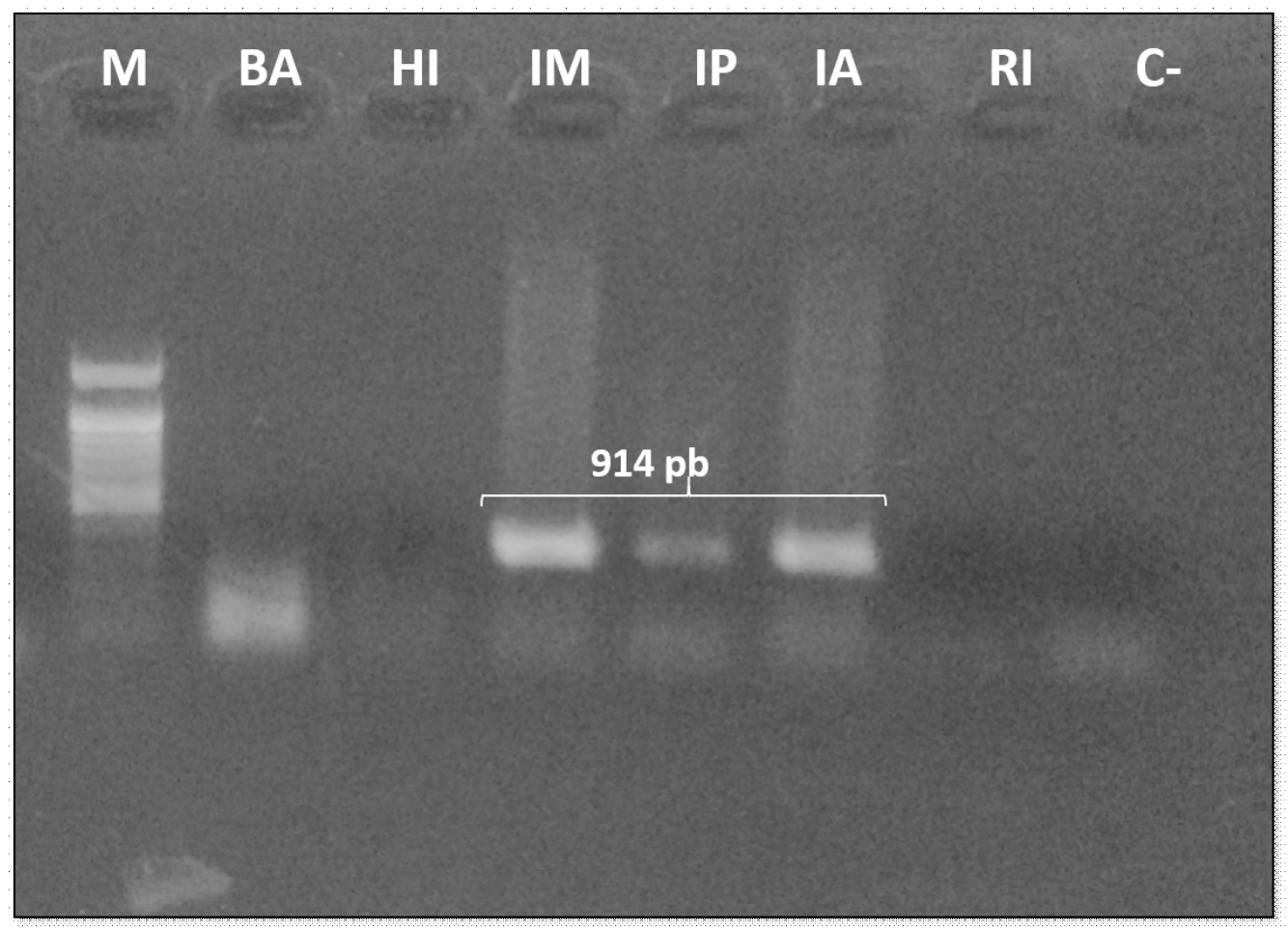

Figura 2. Distribución del PepT1 en tejidos del paiche con los iniciadores F3, R1. M: Marcador de peso molecular de $100 \mathrm{pb}$ (Thermofisher); BA: Bazo; HI: Hígado; IM: Intestino medio; IP: Intestino posterior; IA: Intestino anterior; RI: Riñón; C-: Control negativo

de modificación postraduccional o de otro tipo (Kusman et al., 2006; Wright et al., 2011). Los hallazgos del presente estudio a través de la espectrometría de masas MALDI TOF/ TOF confirmaron la presencia de la proteína transportadora de péptidos (PepT1) al obtener secuencias peptídicas homólogas al PepT1 de varias especies de peces. Esta técnica fue utilizada por Zhang et al. (2016) quienes identificaron proteínas diferenciales en las vellosidades y criptas del intestino entre ratones knockout y wildtype para el PepT1. Por otro lado, Cueva (2016) utilizó esta herramienta, la que le permitió identificar neurohormonas involucradas en la reproducción, así como identificación de proteínas asociadas a la inmunidad y estrés en paiche, abriendo la vía para futuros trabajos de caracterización de otras proteínas de interés.

\section{Conclusiones}

- Se logró identificar y caracterizar parcialmente la proteína transportadora de péptidos PepT1 en el intestino de paiche A. gigas.

- La identificación del pept 1 mediante la espectrometría de masas MALDI TOF/ TOF puede ser una buena alternativa a los métodos tradicionales, ya que es sensible, y su alta precisión en la medición de las masas y la secuenciación masiva de péptidos reduce los problemas de identificación de proteínas y péptidos.

\section{Agradecimientos}

Los autores agradecen a la empresa Acuicultura de Huaura S.A.C por brindar el material biológico. Al MSc Max Salvatierra 


\section{A. Fragmento 1}

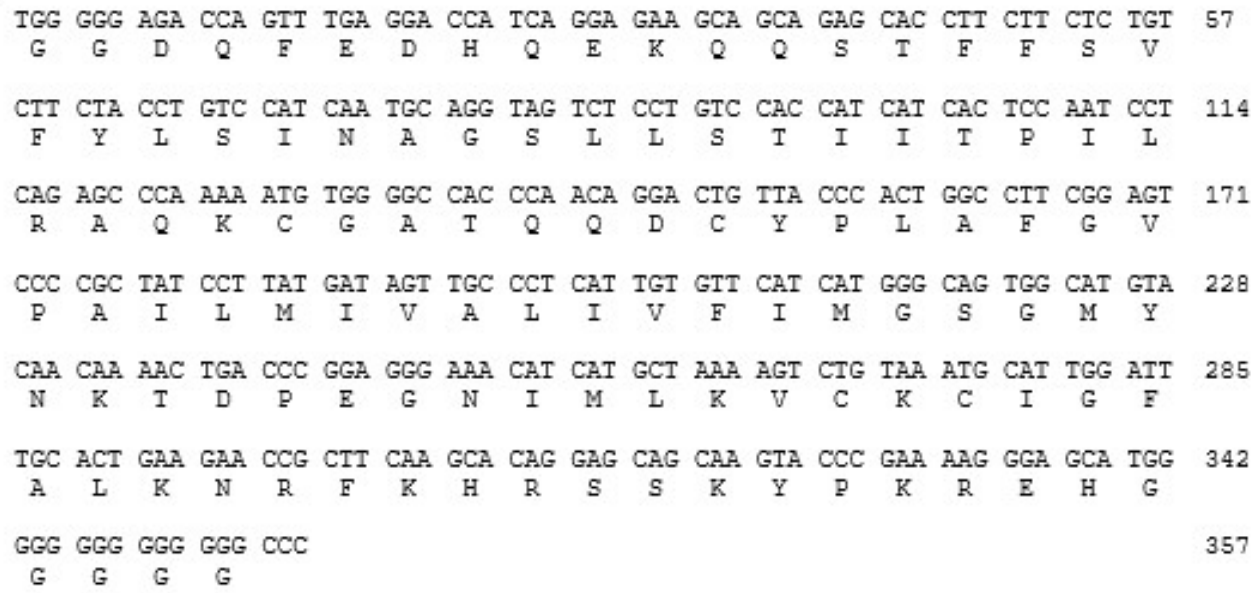

\section{B. Fragmento 2}

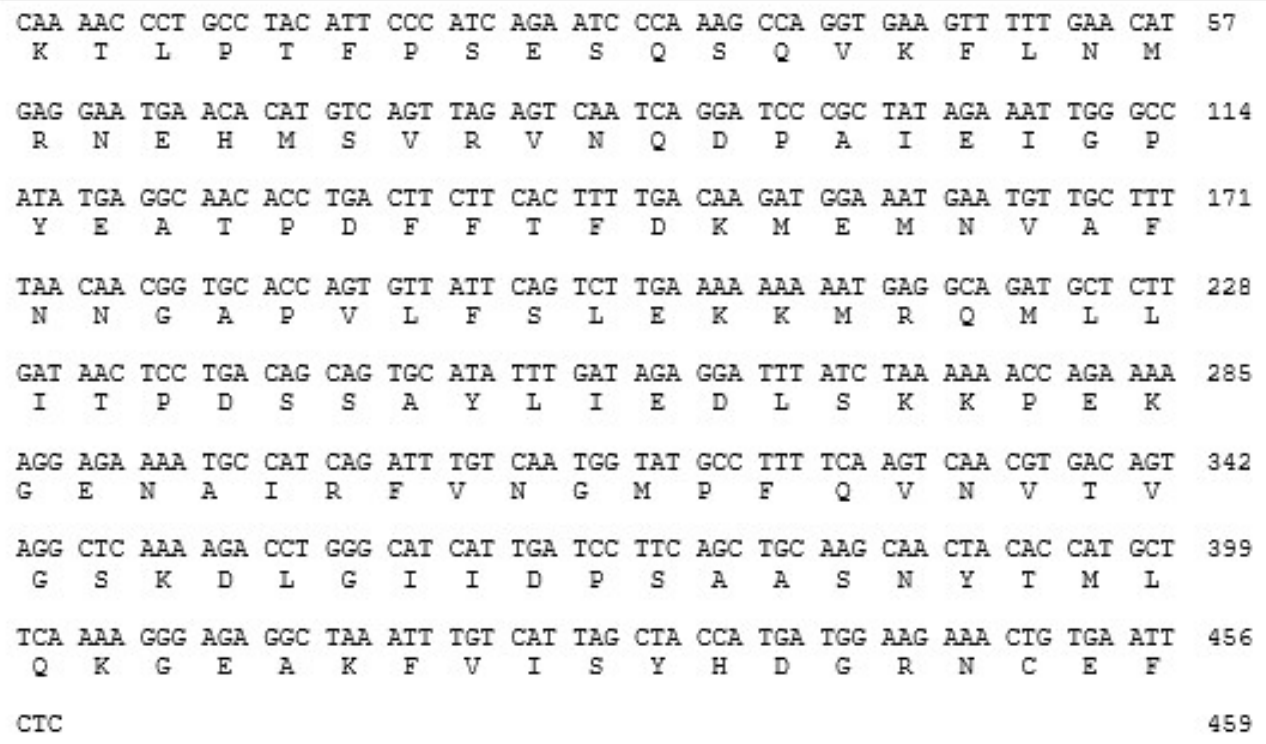

Figura 3. Secuencias de nucleótidos de los fragmentos del PepT1 de paiche divididos en codones, con sus respectivas secuencias de aminoácidos predichos. A: Fragmento 1, amplificado con los iniciadores degenerados PepT1 F3 y PepT1 R1. B: Fragmento 2, amplificado con los iniciadores degenerados PepT1 F2 y PepT1 R3 
Alor por el apoyo brindado. Circulo de investigación en biotecnología molecular para el desarrollo y la sostenibilidad de los sectores acuícolas del Perú (132-2015-FONDECYT.

\section{Literatura Citada}

1. Bakke S, Olderbakk AE, Gómez P, Verri T, Kousoulaki K, Aksnes A, Ronnestad I. 2010. Dietary protein hydrolysates and free amino acids affect the spatial expression of peptide transporter PepT1 in the digestive tract of Atlantic cod (Gadus morhua). Comp Biochem Phys B 156: 48-55. doi: 10.1016/j.cbpb.2010.02.002

2. Bucking C, Schulte P. 2012. Environmental and nutritional regulation of expression and function of two peptide transporter (PepT1) isoforms in a euryhaline teleost. Comp Biochem Phys A 161: 379-387. doi: 10.1016/j.cbpa.2011.12.008

3. Cueva MD. 2016. Aplicación de la espectrometría de masas MALDI TOF/ TOF a la endocrinología, inmunología y patología del paiche Arapaima gigas. Tesis de Magíster. Tumbes, Perú: Univ Nacional de Tumbes. 73 p.

4. Daniel H. 2004. Molecular and integrative physiology of intestinal peptide transport. Annu Rev Physiol 66: 361-384. doi: 10.1146/annurev.physiol.66.032102 .144149

5. [DIDITT] Dirección de Investigación, Innovación, Desarrollo y Transferencia Tecnológica. 2017. Pienso para paiche «Arapaima gigas». Vigilancia tecnológica e inteligencia competitiva. Boletín online N. ${ }^{\circ} 01$. [Internet]. Disponible en: https://www.itp.gob.pe/archivos/ vtic/ACUICULTURA_001-2017.pdf

6. Fernandes JM, Mommens M, Hagen O, Babiak I, Solberg C. 2008. Selection of suitable reference genes for realtime PCR studies of Atlantic halibut development. Comp Biochem Phys B
150: 23-32. doi: 10.1016/j.cbpb.2008.01.003

7. Gatlin DM, Barrows FT, Brown P, Dabrowki K, Gaylord TG, Hardy RW, Hernan E, et al. 2007. Expanding the utilization of sustainable plant products in aquafeeds: a review. Aquac Res 38: 551-579. doi: 10.1111/j.1365-2109.2007. 01704.x

8. Gilbert ER, Wong EA, Webb KE. 2008. Peptide absorption and utilization: implications for animal nutrition and health. J Anim Sci 86: 2135-2155. doi: 10.2527/jas.2007-0826

9. Kiela PR, Ghishan FK. 2016. Physiology of intestinal absorption and secretion. Best Pract Res Cl Ga 30:14559. doi: 10.1016/j.bpg.2016.02.007

10. Liu Z, Zhou Y, Feng J, Lu S, Zhao Q, Zhang J. 2013. Characterization of oligopeptide transporter (PepT1) in grass carp (Ctenopharyngodon idella). Comp Biochem Phys B 164: 194-200. doi: 10.1016/j.cbpb.2012.11.008

11. Liu Z, Zhou Y, Liu S, Zhao Q, Feng J, Lu S, Xiong G, et al. 2014. Characterization and dietary regulation of oligopeptide transporter (PepT1) in different ploidy fishes. Peptides 52: 149156. doi: 10.1016/j.peptides.2013.12.017

12. Oeda AP, Vitti G, Rodrigues V. 2015. Alimentacao e nutricao do pirarucu (Arapaima gigas). EMBRAPA Pesca e Aquicultura. Doc 18. Brasil: EMBRAPA. $26 \mathrm{p}$.

13. Ostaszewska T, Szatkowska I, Verri TP, Dabrowski K, Romano A, Barca I, Muszynksa M, et al. 2009. Cloning two PepT1 cDNA fragments of common carp, Cyprinus carpio [Actinopterygii: Cypriniformes: Cyprinidae] Acta Ichthyol Piscat 39: 81-86. doi: 10.3750/ AIP2009.39.2.01

14. Ostaszewska T, Kamaszewski M, Grochowski P, Dabrowski K, Verri T, Aksakal E, Szatkowska I, et al. 2010. The effect of peptide absorption on PepT1 gene expression and digestive 
system hormones in rainbow trout (Oncorhynchus mykiss). Comp Biochem Phys A 155: 107-114. doi: 10.1016/ j.cbpa.2009.10.017

15. Rebaza M, Rebaza C, Deza S. 2010. Densidad de siembra para cultivos de paiche en jaulas flotantes. Aquavision 6: 26-27.

16. Regina F, Oliveira D, Warlen P, Alves de Costa F, Foncesa V, DalPai M. 2014. Expression of growth-related factors in skeletal muscle of pirarucu (Arapaima Gigas) during growth. J Aquac Res Dev 5: 6. doi: 10.4172/21559546.1000272

17. Romano A, Barca A, Storelli C, Verri T. 2014. Teleost fish models in membrane transport research: the PEPT1 (SLC15A1) H+-oligopeptide transporter as a case study. J Physiol 592: 881-897. doi: 10.1113/jphysiol.2013.259622

18. Ronnestad I, Gavaia PJ, Viegas CS, Verri T, Romano A, Nilsen TO, Jordal AO, et al. 2007. Oligopeptide transporter Pept1 en Atlantic cod (Gadus morhua L.): cloning, tissue expression and comparative aspects. J Exp Biol 210: 3883-3896. doi: 10.1242/ jeb.007898

19. Ronnestad I, Murashita K, Kottra G, Jordal AE, Narawane S, Jolly C, Daniel H, et al. 2010. Molecular cloning and functional expression of Atlantic salmon peptide transporter 1 in Xenopus oocytes reveals efficient intestinal uptake of lysine-containing and other bioactive di- and tripeptides in teleost fish. J Nutr doi: 10.3945/jn.109.118240

20. Ronnestad I, Akiba Y, Kaji I, Kaunitz JD. 2014. Duodenal luminal nutrient sensing. Curr Opin Pharmacol 19: 6775. doi: 10.1016/j.coph.2014.07.010

21. Sambrook J, Russell D. 2001. Molecular cloning. A laboratory manual. New York, USA: Cold Spring Laboratory Press. 999 p.

22. Segura M, Chel L, Betancur D. 2010. Effect of digestion on bioavailability of- peptides with biological activity. Rev Chil Nutr 37: 386-391. doi: 10.1080/ 87559129.2011.563395

23. Shen H, Smith DE, Brosius FC. 2001. Developmental expression of PEPT1 and PEPT2 in rat small intestine, colon, and kidney. Pediatr Res 49: 789-795. doi: 10.1203/00006450-200106000-00013

24. Shevchenko A, Tomas H, Havlis J, Olsen J, Mann M. 2007. In-gel digestion for mass spectrometric characterization of proteins and proteomes. Nat Protocols 1: 2856-2860. Doi: 10.1038/ nprot.2006.468

25. Storelli C, Vilella S, Romano MP, Maffia M, Cassano G. 1989. Brushborder amino acid transport mechanisms in carnivorous eel intestine. Am J Physiol 257: 506-510 doi: 10.1152/ajpregu.1989.257.3.R506

26. Su Y, Zhang R, Dong J, Yang C. 2011. Evaluation of internal control genes for qRT-PCR normalization in tissues and cell culture for antiviral studies of grass carp (Ctenopharyngodon idella). Fish Shellfish Immun 30: 830-835. doi: 10.1016/j.fsi.2011.01.006

27. Terova G, Corá S, Verri T, Rimoldi S, Bernardini G, Saroglia M. 2009. Impact of feed availability on PepT1 mRNA expression levels in sea bass (Dicentrarchus labrax). Aquaculture 294: 288-299. doi: 10.1016/j.aquaculture.2009.06.014

28. Thamotharan M, Gomme J, Zonno V, Maffia M, Storelli C, Ahearn GA. 1996. Electrogenic, proton-coupled, intestinal dipeptide transport in herbivorous and carnivorous teleosts. Am J Physiol 270: 939- 947. doi: 10.1152/ajpregu.1996.270.5.R939

29. Verri T, Kottra G, Romano A, Tiso N, Peric M, Maffia M, Boll M, et al. 2003. Molecular and functional characterisation of the zebrafish (Danio rerio) PEPT1type peptide transporter. Febs Lett 549: 115-116. doi: 10.1016/S0014-5793(03)00759-2 
30. Verri T, Terova G, Dabrowski K, Saroglia M. 2011. Peptide transport and animal growth: the fish paradigm. Biology Lett 7: 597-600. doi: 10.1098/rsbl.2010.-1164

31. Wang J, Yan $X$, Lu R, Meng $X$, Nile G. 2017. Peptide transport 1 (Pept1) in fish: a review. Aquac Fisheries 2: 193206. doi: 10.1016/j.aaf.2017.06.007

32. Wright PC, Noirel J, Ow S, Fazeli A. 2011. A review of current proteomics technologies with a survey on their widespread use in reproductive biology investigations. Theriogenology 77: 738-765. doi: 10.1016/j.theriogenology.-2011.11.012

33. Zhang Y, Viennois E, Zhang M, Xiao, Kwon M, Walter L, Garg P, et al. 2016. PepT1 Expression helps maintain intestinal homeostasis by mediating the differential expression of miRNAs along the crypt-villus axis. Sci Rep 6: 27119. doi: 10.1038/srep27119 